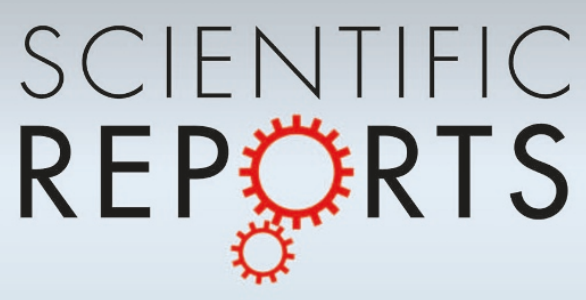

OPEN

SUBJECT AREAS:

BATTERIES

ELECTRONIC PROPERTIES AND

DEVICES

CHEMICAL ENGINEERING

SYNTHESIS AND PROCESSING

Received

11 April 2013

Accepted

12 August 2013

Published

3 September 2013

Correspondence and requests for materials should be addressed to

A.L.M.R. (leela@rice. edu) or P.M.A.

(ajayan@rice.edu)

\section{Supercapacitor Operating At 200 Degrees Celsius}

\author{
Raquel S. Borges 1,2, Arava Leela Mohana Reddy' , Marco-Tulio F. Rodrigues ${ }^{1,2}$, Hemtej Gullapalli', \\ Kaushik Balakrishnan', Glaura G. Silva'2 \& Pulickel M. Ajayan'
}

'Department of Mechanical Engineering and Materials Science, Rice University, Houston, TX-77005, ²Depto de Química, Universidade Federal de Minas Gerais (UFMG), $31270-901$ Belo Horizonte, Brazill.

The operating temperatures of current electrochemical energy storage devices are limited due to electrolyte degradation and separator instability at higher temperatures. Here we demonstrate that a tailored mixture of materials can facilitate operation of supercapacitors at record temperatures, as high as $200^{\circ} \mathrm{C}$. Composite electrolyte/separator structures made from naturally occurring clay and room temperature ionic liquids, with graphitic carbon electrodes, show stable supercapacitor performance at $200^{\circ} \mathrm{C}$ with good cyclic stability. Free standing films of such high temperature composite electrolyte systems can become versatile functional membranes in several high temperature energy conversion and storage applications.

here have been several innovations in the electrochemical storage area during the last decade to address the critical demands of power delivery ${ }^{1-8}$. Recently new materials such as graphene and its derivatives have been used as electrodes in applications such as supercapacitors and batteries ${ }^{9-12}$. Of the several issues being addressed for electrochemical energy storage devices, high temperature capabilities for powering sensors in oil drilling industries (downhole temperatures are above $120^{\circ} \mathrm{C}$ ), military and space applications has not yet fully addressed. The limiting factor for these device to work at high temperature is the stability of the electrolyte ${ }^{13}$. The conventional aqueous electrolytes, though easy to handle, cannot sustain high temperatures ${ }^{2,14}$. The use of organic electrolytes, dissolved in organic solvents such as acetonitrile ${ }^{15}$ with low boiling points $\left(<80^{\circ} \mathrm{C}\right)$, limit them for use at higher temperatures ${ }^{13}$. Room-temperature ionic liquids (RTILs) have been considered as alternative electrolytes to address the issue of high temperature stability ${ }^{16}$. RTILs have several advantages such as low vapor pressure, non-flammability, thermal stability, low toxicity and large electrochemical potential window. The drawback of using them in conventional devices is their lower ionic conductivity at lower temperatures, due to their high viscosity. As RTIL's have higher thermal stability, at elevated temperatures, they become less viscous and hence have better ionic conductivity. This makes them apparent candidates for high temperature energy devices and have already been used in devices up to $100^{\circ} \mathrm{C}^{13}$. Though RTIL's can withstand much higher temperatures, another component of the energy storage device, the separator, which encapsulates the electrolyte and acts as the ion-permeable membrane separating the electrodes limits the temperature stability ${ }^{17}$. Several separators such as cellulose papers, cellophane fabrics, polymers, asbestos, glass wool etc., have been used for ambient temperature operations but are not reliable at higher temperatures as they shrink at elevated temperatures and leads to electrical shorts in the device. Some advances have combined the electrolyte and separator into a single component, by developing a family of solid and gelled electrolytes ${ }^{18-22}$. None of the above however has been shown to operate above $100^{\circ} \mathrm{C}$ with good cyclic stability. Here, we demonstrate a unique composite separator/ electrolyte membrane, consisting of RTIL and naturally occurring clay material, resulting in excellent thermal stability and good ionic conductivity allowing energy storage devices to perform stably up to $200^{\circ} \mathrm{C}$ with good cycling capabilities.

\section{Results}

First, in order to use RTIL for high temperature applications, its thermal inertness during cycling and its ionic conductivity are critical; therefore, BMMI-TFSI was chosen as electrolyte for this study ${ }^{23-26}$. Ionic conductivity as a function of temperature was studied by conducting electrochemical impedance spectroscopy measurements from room temperature to $100^{\circ} \mathrm{C}$. Ionic conductivity of BMMI-TFSI is observed to be in the order of $\mathrm{mS} / \mathrm{cm} \mathrm{and}$ increases with increase of temperature; the trend is consistent with other reported works (see supporting information figure $\mathrm{S} 1)^{25,26}$. A supercapacitor standard test cell was fabricated using BMMI-TFSI as an electrolyte, celgard separator ( $\sim 20 \mu \mathrm{m}$ thick) and RGO as electrodes. RGO was obtained by chemical reduction of graphite 
oxide (GO) and coated on to stainless steel current collectors (see supporting information for characterization; Figure S2 and S3). The electrochemical properties of the test cell were investigated by cyclic voltammetry $(\mathrm{CV})$ and galvanostatic charge-discharge $(\mathrm{CD})$ measurements. Recorded cyclic voltammograms at different temperatures (room temperature and $100^{\circ} \mathrm{C}$ ) are shown in supporting information Figure S4. Stable and rectangular shapes of the voltammogram, symmetric in anodic and cathodic directions, at different temperatures were observed at scan rate of $60 \mathrm{mV} . \mathrm{s}^{-1}$. The increase in area of the CV curve with increase of temperature indicates an enhancement in the specific capacitance, which can be attributed to effective utilization of electrode material by the electrolyte and also its improved ionic conductivity. Specific capacitance reached values greater than $70 \mathrm{~F} / \mathrm{g}$ at $100^{\circ} \mathrm{C}$. Galvanostatic charge-discharge measurements were conducted at room temperature and $100^{\circ} \mathrm{C}$, and the resultant voltage $v s$ time curves for the RTIL electrolyte based supercapacitor at $100^{\circ} \mathrm{C}$ is shown in supporting information Figure S4. The results are in agreement with literature using ionic liquids as electrolyte ${ }^{27}$, however shows a lower capacitance than other reports that use addition of solvents to decrease the ionic liquid viscosity ${ }^{9}$.

These supercapacitors with RTIL electrolyte and celgard separators had good performance as expected until $100^{\circ} \mathrm{C}$ but they cannot withstand temperatures any higher. To improve the thermal stability of the system, naturally occurring Bentonite clay is mixed with the RTIL and used as a separator/electrolyte composite membrane (see supporting information Figures S5-S8 for the characterization of the clay and the clay:RTIL materials). Figure 1A shows the schematic depiction of the operating principle and individual components of Clay:RTIL electrolyte based supercapacitor device. Bentonite clay (from Southern Clay Products) has $99 \%$ purity with platelet size of 500-1000 nanometers across and 1 nanometer thick. A homogenous mixture of this clay and RTIL, 1-Butyl-2,3-dimethylimidazolium bis(trifluoromethylsuphonyl)imide (BMMI-TFSI) was prepared and coated on to a reduced graphene oxide (RGO) electrode. Figure 1B shows the scanning electron micrographs of the interface a contiguous one, between the electrode and the separator/electrolyte. The RTIL infused clay is thermally inert and does not show any visual change upon heating until $200^{\circ} \mathrm{C}$ as seen from the optical images in Figure 1C. The Photographs were taken one hour after samples reached the indicated temperature, color variation is observed only after heating it to $300^{\circ} \mathrm{C}$.

Electrochemical and thermal properties of BMMI-TFSI were characterized to validate its candidature. Absence of any noticeable electrochemical reduction and oxidation reactions between $-3.0 \mathrm{~V}$ to $+3.0 \mathrm{~V}$ from the cyclic voltametry measurement seen in Figure 2A indicates that BMMI-TFSI can be successfully employed for $6 \mathrm{~V}$ electrochemical window applications; this result is in agreement with previous reports ${ }^{23,24}$. The optimum composition of the mixture was deduced by measuring the temperature stability and ionic conductivity at various ratios of clay to RTIL. A $1: 1(\mathrm{w} / \mathrm{w})$ ratio was observed to have the best performance. To confirm the thermal stability of the composite, thermogravimetric measurements were conducted on clay alone, RTIL alone and $1: 1(\mathrm{w} / \mathrm{w})$ of clay:RTIL composite and compared in Figure 2B. It clearly shows that clay:RTIL composite can withstand up to $300^{\circ} \mathrm{C}$ without any significant mass degradation in air. Further, in order to understand the effect of temperature on ionic conductivities of clay:RTIL composite, impedance spectroscopy measurements were conducted at various temperatures (see supporting information Figure S8 and S9). The ionic conductivity of the composite increases almost linearly until $180^{\circ} \mathrm{C}$ and then saturates at $200^{\circ} \mathrm{C}$ (Figure 2C). For comparison, ionic conductivity of pristine RTIL is also shown along with clay:RTIL composite. The slight decrease in ionic conductivity of clay:RTIL composite as compared to pristine RTIL at all temperatures can be attributed to the clay induced higher viscosity for the electrolyte.

Having confirmed that the clay:RTIL composite membrane can survive temperatures until $200^{\circ} \mathrm{C}$ without compromising on the ionic conductivity, supercapacitor devices were fabricated using this composite membrane as the electrolyte/separator. The supercapacitor device in the configuration RGO/clay:RTIL/RGO is made with symmetric electrodes. The performance of the device was tested by conducting cyclic voltammograms measurements at different temperatures RT, 120 and $200^{\circ} \mathrm{C}$ (Figure 3A). The observed stable cyclic voltammograms at $200^{\circ} \mathrm{C}$ clearly indicates the successful formation of double layer within the electrodes without any electrical short
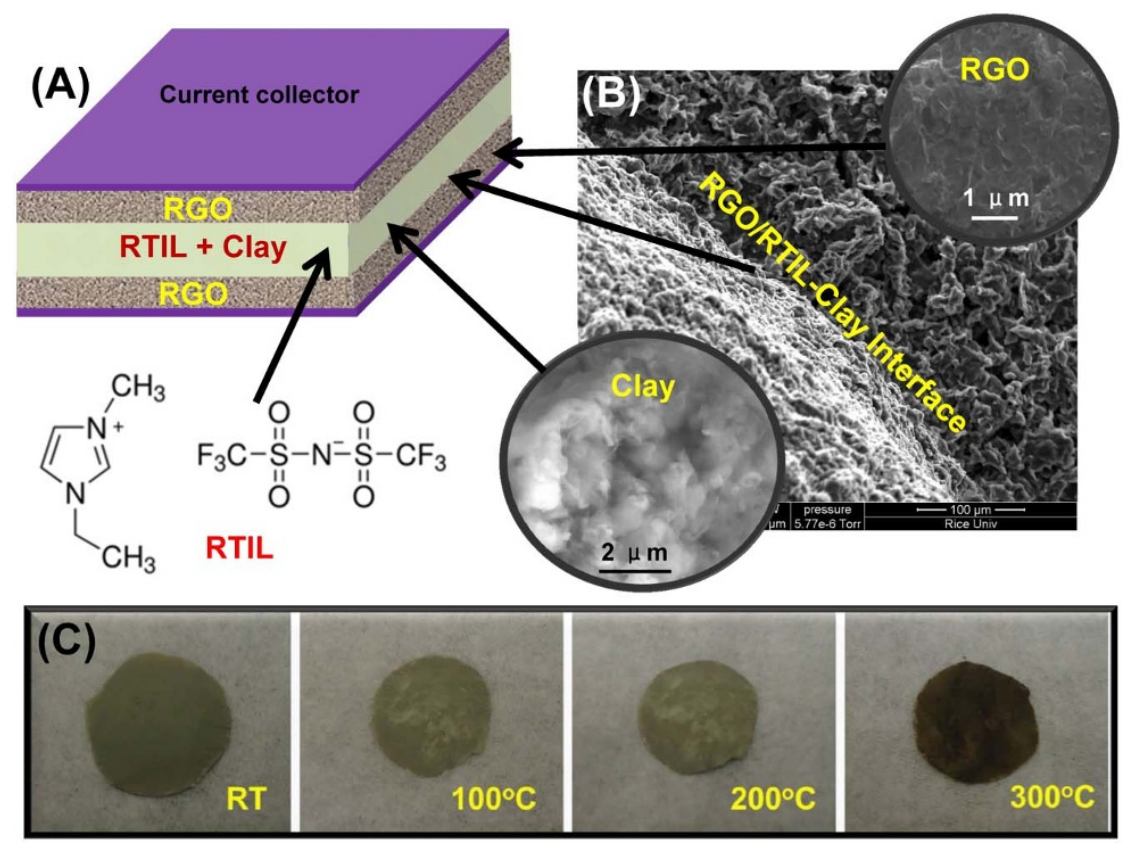

Figure $1 \mid$ (A) Schematic depiction of the operating principle and individual components of Clay:RTIL electrolyte based supercapacitor device, (B) SEM micrograph of RGO/clay:RTIL half capacitor configuration showing seamless interface between electrode/electrolyte; SEM images of clay and RGO can be seen in individual insets (C) Optical pictures of the clay based electrolytes after treatment in different temperatures. 

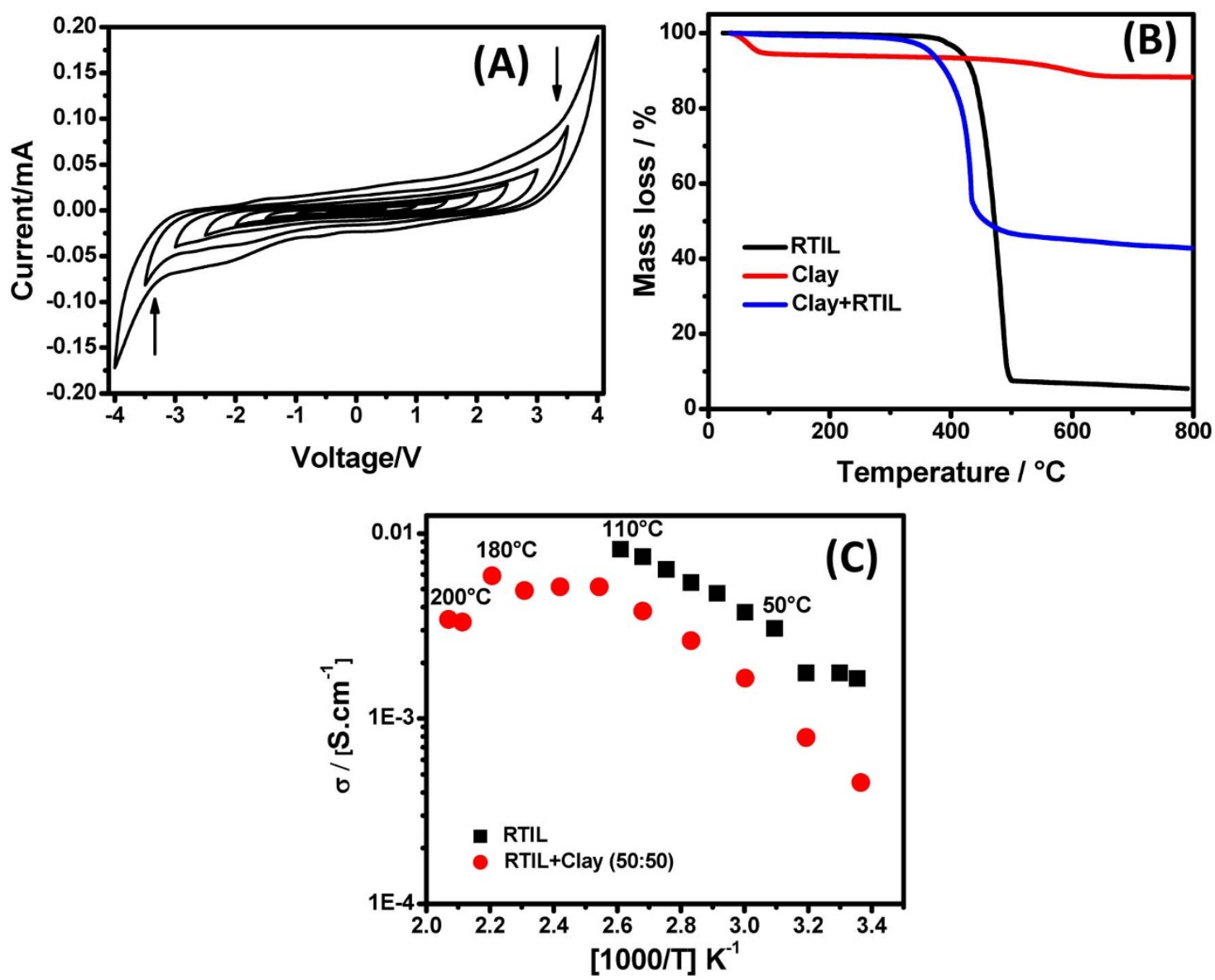

Figure $2 \mid$ (A) Cyclic voltammograms for the room temperature ionic liquids (BMMI-TFSI) using different voltage windows at $50 \mathrm{mV} . \mathrm{s}^{-1}$ and stainless steel current collector. The RTIL shows stable behavior without any significant reaction between $-3 \mathrm{~V}$ and $+3 \mathrm{~V}$. (B) Termogravimetric curves for RTIL (black), Clay (red) and Clay:RTIL (blue) using $10^{\circ} \mathrm{C} / \mathrm{min}$ heating rate. (C) Arrhenius plot for 1-Butyl-2,3-dimethylimidazolium

bis(trifluoromethylsulfonyl)imide and for the mixture Clay:RTIL $(1: 1)$.

circuit. The device can be operated up to a $2.5 \mathrm{~V}$ positive potential window without exhibiting significant pseudo-capacitance, however, the observed side reactions that can take place at high temperature and larger potential window could be due to the decomposition of trapped moisture in the clay system. Specific capacitance $v s$ cycle number plots extracted from this data are presented in Figure 3B. It is seen that although there is a slight drop during the initial cycles, the device has a stable specific capacitance of $20 \mathrm{~F} / \mathrm{g}$ and $40 \mathrm{~F} / \mathrm{g}$ in $0-2 \mathrm{~V}, 0-2.5 \mathrm{~V}$ respectively even after 10,000 cycles of charge-discharge. The observed initial drop in the specific capacitance can be attributed to irreversible solid electrolyte interface reactions, a commonly observed phenomenon ${ }^{28}$. It is observed that energy density and power density of RGO/clay: RTIL/RGO supercapacitor increases with the increase of temperature. Figure 3C shows energy density vs power density plot of $\mathrm{RGO} /$ clay:RTIL/RGO supercapacitor with variation in temperature and voltage window. Two orders of magnitude increase in the power density of the supercapacitor observed at $200^{\circ} \mathrm{C}$ compared to room temperature is attributed to the significant increase in the ionic conductivity of the electrolyte at higher temperature. Self-discharge characteristics of these devices were also studied at various temperatures. The devices were charged to $2 \mathrm{~V}$ with a constant current of $10 \mu \mathrm{A}$ and the voltage was held for 2 hours with a variable current and then allowed to undergo self-discharge with open terminals. Voltage was measured every 2 minutes and it is observed that the devices retained more than half their initial voltage even after 7 hours and $200^{\circ} \mathrm{C}$ (Figure 3D).
In order to increase the versatility of using the above separator/ electrolyte system, it would be easier to use a free standing film which can be cut into different shapes and sizes and inserted into any device. With further motivation of developing such a free standing membrane analogous to the Nafion membranes (commonly used in fuel cells), we added 10\% (by weight) of Themoplastic poly(urethane) (TPU) to the clay:RTIL mixture. The composition was then cast into a film and peeled out, to form a free standing film as shown in Figure 4A. Ionic conductivities of this membrane (TPU:Clay:RTIL) as a function of temperature are extracted from electrochemical impedance measurements and shown in Figure 4B. The membrane is seen to have superior conductivity at higher temperatures, about two orders more at $200^{\circ} \mathrm{C}$, than at room temperature. The addition of polymer to the clay:RTIL mixture reduces the conductivity at room temperature, but at elevated temperatures, the loss is minimal as seen from Figure 4B. We fabricated a supercapacitor device using RGO electrodes and TPU:Clay:RTIL membrane and studied its electrochemical properties. Recorded CVs at different temperatures over a 5.0 V electrochemical window are shown in Figure 4C. Again, the increase in area under CV curves is an indication of the enhanced capacitance at higher temperatures. Galvanostatic charge-discharge measurements were conducted at different temperatures, different potential widows and also at different current rates. Figure 4D summarizes the effect of temperature, potential window and applied current rates on the specific capacitance of the membrane based supercapacitor. A maximum specific capacitance of $33 \mathrm{~F} / \mathrm{g}$ has been observed at $200^{\circ} \mathrm{C}$ for this membrane based supercapacitor. 

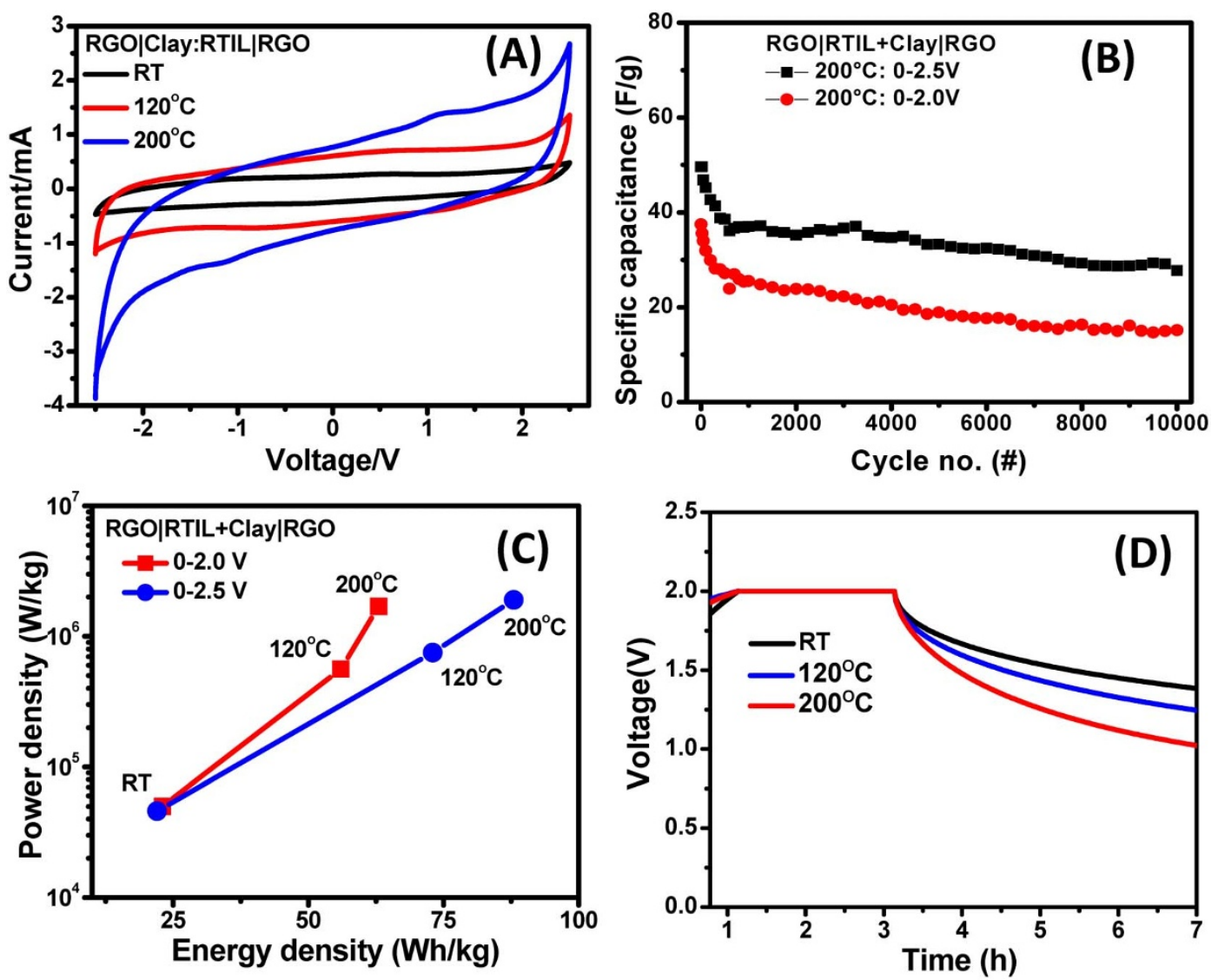

Figure 3 | (A) Cyclic voltammograms for supercapacitor based on clay:RTIL electrolyte at $60 \mathrm{mV} \cdot \mathrm{s}^{-1}$. The capacitor exhibits a box-like shape using a potential window of $5 \mathrm{~V}$ at different temperatures: RT, $120^{\circ} \mathrm{C}$ and $200^{\circ} \mathrm{C}$. (B) Cycling stability of the capacitor RGO $\mid \mathrm{Clay}: \mathrm{RTIL} / \mathrm{RGO}$ at $200^{\circ} \mathrm{C}$ with different voltage windows $2 \mathrm{~V}$ and $2.5 \mathrm{~V}$. (C) Energy density vs power density of the capacitor RGO $\mid$ Clay:RTIL $\mid$ RGO at different temperatures RT, $120^{\circ} \mathrm{C}$ and $200^{\circ} \mathrm{C}$ with variation in voltage windows $2.0 \mathrm{~V}$ and $2.5 \mathrm{~V}$. (D) Self-discharge profiles of the supercapacitor based on clay:RTIL electrolyte after charging to $2 \mathrm{~V}$ at a constant current, holding the voltage at $2 \mathrm{~V}$ by supplying a variable current for 2 hours and then allowing the device to self-discharge. To minimize the influence of measuring instruments, measurements were taken at a time interval of 2 minutes.

\section{Discussion}

The exchangeable cations between the tetrahedral layers of the clay can be mobilized with RTIL, 1-Butyl-2,3-dimethylimidazolium bis (trifluoromethylsuphonyl)imide (BMMI-TFSI), as shown in some reports where small cations existing between layers of clay were replaced by different ions ${ }^{29-31}$. In our case the mechanical grinding process to make the RTIL-clay mixture was designed to obtain a homogeneous electrolyte paste so as to facilitate good ion mobilization between the clay layers. As a result, the clay-RTIL composite performs a dual role of an electrolyte and a separator in the supercapacitor device, by providing ions to the reduced graphite oxide (RGO) symmetric electrodes while still electrically separating. It is observed that ionic conductivity of Clay:RTIL system enhances by three orders of magnitude as we move from room temperature to $200^{\circ} \mathrm{C}$. This is mainly due to decrease in viscosity of RTIL at higher temperatures and hence resulted in better ionic conductivity; this is direct consequences of enhanced power density of supercapacitor at higher temperature.

From self-discharge characteristics of these devices, it is observed that these devices retained more than half their initial voltage even after 7 hours at $200^{\circ} \mathrm{C}$. In order to have such good charge retention at higher temperatures, leakages in electrode material and also the interfaces between various components should be minimized. For RGO synthesis, high degree of reduction was achieved and was confirmed using thermogravimetric measurements (TG). RGO was coated uniformly on to current collectors and the resultant electrodes showed a conductivity of $3 \mathrm{mS} \mathrm{cm}{ }^{-1}$ at room temperature.
To summarize, the high temperature electrochemical energy storage concept has been realized through developing a stable separator/ electrolyte composite. Operating temperature of up to $200^{\circ} \mathrm{C}$ for supercapacitors made using this composite has been demonstrated, owing to the high thermal stability of clay in the composite. The performance of these devices is found to be superior at higher temperatures due to increased ionic mobility of RTIL present in the composite. To facilitate ease of manufacturing a flexible polymer added membrane was also developed out of the clay-RTIL. The high temperature supercapacitor device built using RGO electrodes and clay-RTIL membrane electrolyte shows nearly twice an increase in the operational temperature range compared to any existing devices of our knowledge. The present work could provide solutions for several high temperature energy storage problems.

\section{Methods}

Characterization of 1-Butyl-2,3-dimethylimidazolium bis(trifluoromethylsulfonyl)imide. The room temperature ionic liquid from Iolitec presents $99 \%$ purity and water content of less than $100 \mathrm{ppm}$, according to the supplier. Handling of the ionic liquid was carried out in a glove box Unilab MBraun under argon atmosphere, with water level less than $0.1 \mathrm{ppm}$ and oxygen level less than $10 \mathrm{ppm}$.

Electrochemical properties of the room temperature ionic liquid (BMMI-TFSI) were measured by cyclic voltammetry and electrochemical impedance spectroscopy (EIS). Electrical measurements were performed in an AUTOLAB PGSTAT $302 \mathrm{~N}$ ECOCHEMIE frequency analyzer. Cyclic voltammetry measurements were performed in different potential windows ranging from $1 \mathrm{~V}$ to $8 \mathrm{~V}$ with scan rate of $50 \mathrm{mV} \mathrm{s}^{-1}$, a stainless steel current collector was used. EIS experiments were conducted using a frequency range from $0.5 \mathrm{MHz}$ to $0.5 \mathrm{~Hz}$ at $0 \mathrm{~V}$ with amplitude of $10 \mathrm{mV}$. Thermal stability of ionic liquid was obtained by thermogravimetric (TG) measurements performed in a TA Instruments SDT 2960 in air atmosphere at a 

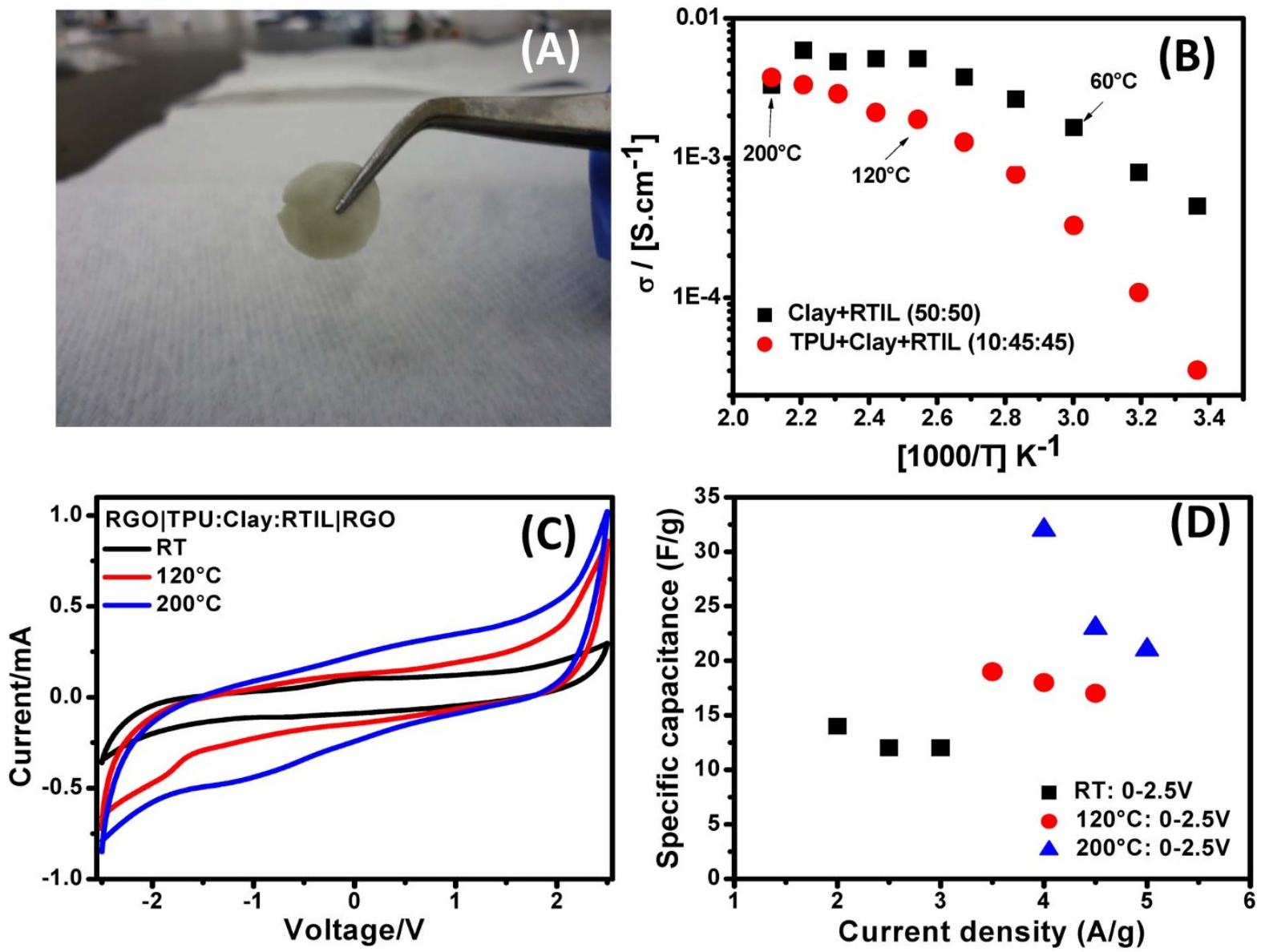

Figure $4 \mid$ (A) Free standing membrane based on TPU:Clay:RTIL working as electrolyte/separator for supercapacitor until $200^{\circ} \mathrm{C}$. (B) Arrhenius plot for the mixture Clay:RTIL and free standing membrane. (C) Cyclic voltammograms for supercapacitor based on RGO electrodes and free standing membrane as electrolyte. The measurements were conducted at room temperature, $120^{\circ} \mathrm{C}$ and $200^{\circ} \mathrm{C}$, using $60 \mathrm{mV} . \mathrm{s}^{-1} \mathrm{scan}$ rate. (D) Specific capacitance as a function of temperature and current density for the capacitor using a membrane as electrolyte. A broad range of current density was applied without loss in capacitor behavior.

heating rate of $10^{\circ} \mathrm{C} \mathrm{min}^{-1}$. Approximately $15 \mathrm{mg}$ of RTIL was used in TG measurements.

Characterization of bentonite clay. The bentonite clay (from Southern Clay Products) has $99 \%$ purity, according to the supplier. Chemical structure of the clay was analyzed by Infrared and Raman measurements. The FTIR spectrum was made in a Perkin-Elmer BX spectrometer, in the Transmission mode. The sample was first dispersed in $\mathrm{KBr}$ and compressed to a compact pellet. The spectrum was acquired after 64 scans with a $4 \mathrm{~cm}^{-1}$ resolution. Micro-Raman experiments were made in a DILOR XY spectrometer using an OLYMPUS BH-2 optical microscope, with a $100 \times$ objective. The excitation in $514.5 \mathrm{~nm}$ with $3 \mathrm{~mW}$ of power was provided by an $\mathrm{Ar}-\mathrm{Kr}$ laser. X-Ray diffraction (XRD) measurements were conducted in a Simens-D5000 diffractometer using a cooper tube and a scan rate of $4^{\circ} \mathrm{C} \mathrm{min}^{-1}$. The morphology of bentonite clay was observed by scanning electron microscopy (SEM). Images were obtained in a FEI QUANTA $20{ }^{\circledR}$ scanning electron microscope using secondary electrons, without any cover over the samples. Thermal properties of bentonite clay were obtained by thermogravimetric (TG) measurements performed in a TA Instruments SDT 2960 in air atmosphere at a heating rate of $10^{\circ} \mathrm{C} \mathrm{min}^{-1}$ Approximately $15 \mathrm{mg}$ of bentonite clay was used in TG measurements.

Fabrication and characterization of Clay:RTIL electrolytes. The new composite electrolyte based on bentonite clay and RTIL was prepared mixing appropriate ratio of each material using a mortar until obtain a homogenous paste like electrolyte. This electrolyte was spread onto a stain steel current collector in order to perform electrochemical measurements, cyclic voltammetry and electrochemical impedance spectroscopy (EIS). Electrical measurements were performed in an AUTOLAB PGSTAT 302 N ECOCHEMIE frequency analyzer. Cyclic voltammetry

measurements were performed in different potential windows ranging from $1 \mathrm{~V}$ to $7 \mathrm{~V}$ with scan rate of $50 \mathrm{mV} \mathrm{s}^{-1}$. EIS experiments were conducted using a frequency range from $0.5 \mathrm{MHz}$ to $0.5 \mathrm{~Hz}$ at $0 \mathrm{~V}$ with amplitude of $10 \mathrm{mV}$. Thermogravimetric (TG) measurement of clay:RTIL electrolyte was performed in a TA Instruments SDT

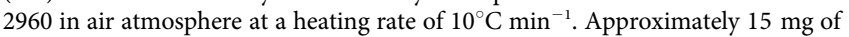
electrolyte was used in TG measurements. Chemical structure of washed clay:RTIL composite was analyzed by Infrared measurement, the composite was prepared as described above, then washed at least ten times using distilled water to eliminate RTIL that was adsorbed in excess on clay structure. The sample was dried at $100^{\circ} \mathrm{C}$ for several hours, then dispersed in $\mathrm{KBr}$ and finally compressed to a compact pellet. The FTIR spectrum was made in a Perkin-Elmer BX spectrometer, in the Transmission mode. The spectrum was acquired after 64 scans with a $4 \mathrm{~cm}^{-1}$ resolution. The morphology of clay:RTIL was observed by scanning electron microscopy (SEM). Images were obtained in a FEI QUANTA $200{ }^{\circledR}$ scanning electron microscope using secondary electrons, without any cover over the samples.

Fabrication and characterization of TPU:Clay:RTIL electrolytes. The new electrolyte membrane based on a thermoplastic polyurethane (Irogran PS455-203 from Huntsman), bentonite clay and RTIL was prepared mixing a paste like electrolyte (clay:RTIL) prepared previously with $10 \mathrm{wt} \%$ of polyurethane diluted in tetrahydrofuran (THF). This electrolyte solution was coated on a stain steel current collector in order to evaporate the solvent and obtain an electrolyte film. The electrochemical performance was measured by electrochemical impedance spectroscopy (EIS). Electrical measurements were performed in an AUTOLAB PGSTAT $302 \mathrm{~N}$ ECOCHEMIE frequency analyzer. EIS experiments were conducted using a frequency range from $0.5 \mathrm{MHz}$ to $0.5 \mathrm{~Hz}$ at $0 \mathrm{~V}$ with amplitude of $10 \mathrm{mV}$.

Fabrication and characterization of RGO electrodes. Reduced graphite oxide (RGO) powder was obtained from hydrazine reaction using graphite oxide (GO) powder as precursor. GO was synthesized using Improved Hummer Method (1) from a commercial graphite (Bay Carbon). Thermogravimetric (TG) measurements of GO and RGO were performed in a TA Instruments SDT 2960 in air atmosphere at a heating rate of $5^{\circ} \mathrm{C} \mathrm{min}^{-1}$. Approximately $10 \mathrm{mg}$ of sample was used in TG measurements. RGO ink was prepared in 2-propanol using ultrasonic bath for several hours until obtain a stable dispersion. The electrodes were prepared by drop casting of consecutively layers of RGO dispersion onto stain steel current collector. The morphology of RGO electrodes was analyzed by scanning electron microscopy (SEM). Images were obtained in a FEI QUANTA $200^{\circledR}$ scanning electron microscope using secondary electrons, without any cover over the samples. 
Fabrication and characterization of double layer capacitor using RGO and RTIL. Electrochemical capacitors were prepared in a stacked configuration with two RGO electrodes (prepared directly on stainless steel current collectors) with an ionic liquid layer in-between as an electrolyte. A paper disk separator was used to support the ionic liquid. Electrochemical properties of capacitor were measured by cyclic voltammetry $(\mathrm{CV})$, electrochemical impedance spectroscopy (EIS) and galvanostatic charge-dircharge measurements. Electrical measurements were performed in an AUTOLAB PGSTAT $302 \mathrm{~N}$ ECOCHEMIE frequency analyzer. CV measurements were performed in different potential windows ranging from $1 \mathrm{~V}$ to $4 \mathrm{~V}$ with scan rate of $60 \mathrm{mV} \mathrm{s}^{-1}$. EIS experiments were conducted using a frequency range from $1 \mathrm{MHz}$ to $0.1 \mathrm{~Hz}$ at $0 \mathrm{~V}$ with amplitude of $10 \mathrm{mV}$. Galvanostatic charge-dircharge measurements were obtained at $2.5 \mathrm{~V}$ voltage window, using different current densities.

Fabrication and characterization of high temperature double layer capacitor using a new electrolyte based on Clay:RTIL and based on Clay:RTIL:TPU. Electrochemical capacitors were prepared in a stacked configuration with two RGO electrodes (prepared directly on stainless steel current collectors). A layer of clay:RTIL composite electrolyte (or clay:RTIL:TPU $10 \mathrm{wt} \%$ electrolyte membrane) was spread out in-between RGO electrodes. No separator is necessary in that capacitor configuration. Electrochemical properties of capacitor were measured by cyclic voltammetry $(\mathrm{CV})$, electrochemical impedance spectroscopy (EIS) and galvanostatic charge-dircharge measurements.

1. Gowda, S. R., Leela Mohana Reddy, A., Zhan, X. \& Ajayan, P. M. Building energy storage device on a single nanowire. Nano Lett. 11, 3329-3333 (2011).

2. Simon, P. \& Gogotsi, Y. Materials for electrochemical capacitors. Nat Mater 7, 845-854 (2008).

3. Brezesinski, T., Wang, J., Tolbert, S. H. \& Dunn, B. Ordered mesoporous $\alpha$-MoO3 with iso-oriented nanocrystalline walls for thin-film pseudocapacitors. Nature Materials 9, 146-151 (2010).

4. Aricò, A. S., Bruce, P., Scrosati, B., Tarascon, J.-M. \& van Schalkwijk, W. Nanostructured materials for advanced energy conversion and storage devices. Nat Mater 4, 366-377 (2005).

5. Dunn, B., Kamath, H. \& Tarascon, J.-M. Electrical Energy Storage for the Grid: A Battery of Choices. Science 334, 928-935 (2011).

6. Tarascon, J.-M. \& Armand, M. Issues and challenges facing rechargeable lithium batteries. Nature 414, 359-367 (2001).

7. Miller, J. R. \& Simon, P. MATERIALS SCIENCE: Electrochemical Capacitors for Energy Management. Science 321, 651-652 (2008).

8. Pushparaj, V. L. et al. Flexible energy storage devices based on nanocomposite paper. PNAS 104, 13574-13577 (2007).

9. Miller, J. R., Outlaw, R. A. \& Holloway, B. C. Graphene Double-Layer Capacitor with ac Line-Filtering Performance. Science 329, 1637-1639 (2010).

10. Gao, W. et al. Direct laser writing of micro-supercapacitors on hydrated graphite oxide films. Nat Nano 6, 496-500 (2011).

11. Kumar, A. et al. Direct synthesis of lithium-intercalated graphene for electrochemical energy storage application. ACS Nano 5, 4345-4349 (2011).

12. Gullapalli, H., Mohana Reddy, A. L., Kilpatrick, S., Dubey, M. \& Ajayan, P. M. Graphene Growth via Carburization of Stainless Steel and Application in Energy Storage. Small 7, 1697-1700 (2011).

13. Lin, R. et al. Capacitive Energy Storage from -50 to $100^{\circ} \mathrm{C}$ Using an Ionic Liquid Electrolyte. J. Phys. Chem. Lett. 2, 2396-2401 (2011).

14. Reddy, A. L. M., Shaijumon, M. M., Gowda, S. R. \& Ajayan, P. M. Multisegmented Au-MnO 2/Carbon Nanotube Hybrid Coaxial Arrays for High-Power Supercapacitor Applications. The Journal of Physical Chemistry C 114, 658-663 (2010)

15. Conway, B. E. Electrochemical supercapacitors: scientific fundamentals and technological applications. (Plenum Press, 1999).

16. Armand, M., Endres, F., MacFarlane, D. R., Ohno, H. \& Scrosati, B. Ionic-liquid materials for the electrochemical challenges of the future. Nat Mater 8, 621-629 (2009).
17. Arora, P. \& Zhang, Z. J. Battery separators. Chem. Rev. 104, 4419-4462 (2004).

18. \& Song, J. Y., Wang, Y. Y. \& Wan, C. C. Review of gel-type polymer electrolytes for lithium-ion batteries. Journal of Power Sources 77, 183-197 (1999).

19. Koksbang, R., Olsen, I. I. \& Shackle, D. Review of hybrid polymer electrolytes and rechargeable lithium batteries. Solid State Ionics 69, 320-335 (1994).

20. Aono, H., Imanaka, N. \& Adachi, G. High Li+ Conducting Ceramics. Acc. Chem. Res. 27, 265-270 (1994).

21. Kamaya, N. et al. A lithium superionic conductor. Nat Mater 10, 682-686 (2011).

22. Borges, R. S., Miquita, D. R. \& Silva, G. G. Electrochemical study of double-walled carbon nanotube electrode/block polyether-lithium bis(trifluorosulphonyl)imide salt polymer electrolyte interface. Electrochimica Acta 56, 4650-4656 (2011).

23. Andriyko, Y. O., Reischl, W. \& Nauer, G. E. Trialkyl-Substituted ImidazoliumBased Ionic Liquids for Electrochemical Applications: Basic Physicochemical Properties. J. Chem. Eng. Data 54, 855-860 (2009).

24. Galiński, M., Lewandowski, A. \& Stępniak, I. Ionic liquids as electrolytes. Electrochimica Acta 51, 5567-5580 (2006).

25. Bazito, F. F. C., Kawano, Y. \& Torresi, R. M. Synthesis and characterization of two ionic liquids with emphasis on their chemical stability towards metallic lithium. Electrochimica Acta 52, 6427-6437 (2007).

26. Nadherna, M., Dominko, R., Hanzel, D., Reiter, J. \& Gaberscek, M. Electrochemical Behavior of $\mathrm{Li} 2 \mathrm{FeSiO} 4$ with Ionic Liquids at Elevated Temperature. J. Electrochem. Soc. 156, A619-A626 (2009).

27. Sato, T., Masuda, G. \& Takagi, K. Electrochemical properties of novel ionic liquids for electric double layer capacitor applications. Electrochimica Acta 49, 3603-3611 (2004).

28. Fu, L. J. et al. Surface modifications of electrode materials for lithium ion batteries. Solid State Sciences 8, 113-128 (2006).

29. $\mathrm{Hu}, \mathrm{H}$. et al. Immobilization of ionic liquids in $\theta$-zirconium phosphate for catalyzing the coupling of CO2 and epoxides. RSC Adv. 2, 3810-3815 (2012)

30. Pucci, A., Liuzzo, V., Melai, B., Pomelli, C. S. \& Chiappe, C. Polymerizable ionic liquids for the preparation of polystyrene/clay composites. Polymer International 61, 426-433 (2012)

31. Ha, J. U. \& Xanthos, M. Sequential modification of cationic and anionic nanoclays with ionic liquids. Green Chemistry Letters and Reviews 4, 103-107 (2011).

\section{Acknowledgments}

The authors acknowledge funding support from the Advanced Energy Consortium (AEC). R.S. Borges thanks scholarship from $\mathrm{CNPq}-$ Brazil.

\section{Author contributions}

A.L.M.R. and P.M.A. devised the original concept. A.L.M.R., R.S.B. and H.G. developed the experimental design and performed research. M.F.R., K.B. and G.G.S. contributed to discussions of results. A.L.M.R. and R.S.B. wrote the first draft of the manuscript and all authors participated in manuscript revision.

\section{Additional information}

Supplementary information accompanies this paper at http://www.nature.com/ scientificreports

Competing financial interests: The authors declare no competing financial interests How to cite this article: Borges, R.S. et al. Supercapacitor Operating At 200 Degrees Celsius. Sci. Rep. 3, 2572; DOI:10.1038/srep02572 (2013).

This work is licensed under a Creative Commons AttributionNonCommercial-ShareAlike 3.0 Unported license. To view a copy of this license, visit http://creativecommons.org/licenses/by-nc-sa/3.0 